\title{
LA SIMPLIFICACION DE REGLAS COMO MECANISMO DEL CAMBIO LINGUISTICO: UN EJEMPLO DEL ESPAÑOL DE COSTA RICA
}

\section{Ronald Ross}

Entre los mecanismos del cambio lingüístico que suelen citarse en los estudios tradicionales, la analogía siempre ocupa un lugar prominente. Con cierta frecuencia se ha creído que los efectos de la analogía se limitan a eliminar alomorfos irregulares, haciéndolos conformarse al paradigma normal. En muchos tratados de lingüística tradicional se describe precisamente como una fuerza regularizadora. Sin embargo, este concepto de la analogía es un tanto simplista. Hoenigswald (1963: 40) ha señalado que a veces la analogía crea alomorfos nuevos o generaliza alomorfos irregulares a expensas de sus competidores regulares.

Cuando un cambio analógico reduce el número de casos en que aparece una forma alternante, se dice que ha habido una nivelación analógica. Si en cambio, una modificación analógica multiplica el número de casos en que se emplea una forma altemante, se trata de una extensión analógica.

En el tiempo presente de algunos verbos es-

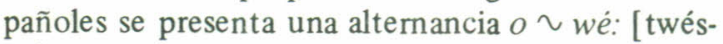
to/tostámos]. No obstante, en la primera conjugación de algunos dialectos ha desaparecido la $O$, de suerte que la "vocal" radical de todo el paradigma pasa a ser el diptongo we. Ya no existe alternancia alguna, por lo que decimos que el paradigma se ha nivelado: [twestár, twestádo, twestándo, twestámos], etc.

Ciertos verbos irregulares de la segunda y tercera conjugaciones añaden a su raíz una consonante velar cuando la vocal siguiente es posterior $(o$ o a): [póngo/póne]. Dialectalmente algunos otros verbos adoptan la misma alternancia, lo cual produce un aumento en el número de formas irregulares: [jwígo, destrwígo]. Cuando esto sucede, nos encontramos ante un caso de extensión analógica. 2. Una lengua es una serie de principios o reglas (léxicas, sintácticas, morfológicas y fonológicas) que relacionan significados con significantes (o una estructura profunda con una estructura superficial). Parece razonable, pues, suponer que un cambio lingüístico (esto es, un cambio en la estructura superficial) es consecuencia directa de una modificación en el sistema de reglas. Son varias las maneras en que las reglas pueden modificarse. Algunas veces se añade al sistema una regla nueva. Otras veces se suprime una antigua. Puede producirse una alteración en el orden de su aplicación $o$, finalmente, es posible que se simplifique una parte de una regla. De particular interés para el presente artículo es la simplificación de reglas por cuanto se cree que corresponde en mayor o menor grado al concepto tradicional de "analogía".

Las reglas de componente morfofonológicol 1 constan de tres partes: el elemento que se va a modificar, la forma como se modifica y el entorno en que se realiza la modificación.

$$
\mathrm{X} \longrightarrow \mathrm{Y} / \mathrm{Z}
$$

Esta regla nos dice que $\mathrm{X}$ se convierte en $\mathrm{Y}$ cuando aparece en el entorno $Z$. La parte de la regla que corresponde al elemento que se va a modificar (X) junto con el entorno en que ocurre el cambio $(\mathrm{Z})$ constituyen el indice estructural. La parte representada por Y se denomina cambio estructural. Cualquiera de estas partes es susceptible de simplificarse, pero las consecuencias no son iguales en ambos casos. Kiparsky (1968: 201) su- 
giere que la simplificación del índice estructural corresponde a la extensión o polarización analógica en tanto que la simplificación del cambio estructural se identifica con la nivelación. Cuando se pierde totalmente el cambio estructural, desaparece la regla y la nivelación es absoluta.

3. A continuación haré unas observaciones con respecto al imperativo español con especial atención a las particularidades morfológicas que presenta en Costa Rica. Me limitaré a las formas del plural las cuales distinguiré unas de otras mediante los rasgos un tanto ad hoc de

A

[ +1 forma $] \quad(=$ comamos $)$

B

B

$\mathrm{C}$

C.

$[+2$ forma $] \quad(=$ comed $)$

[ +3 forma $] \quad(=$ coman $)$

La gramática tradicional objetaría, quizá, que sólo la segunda es un verdadero imperativo y que las otras dos corresponden al subjuntivo. Esta objeción, que se funda más en la gramática del latín que en del español, me parece carente de lógica. Semánticamente es indudable que los tres ejemplos (2) a., b. y c. son imperativos.

\section{2) a. Comámoslo nosotros.}

b. Comedlo vosotros.

c. Cómanlo ustedes.

También existen pruebas sintácticas. En los tres ejemplos tanto el pronombre clítico como el sujeto van pospuestos al verbo. Quienes niegan el carácter imperativo de a. y c. tendrán que explicar de algún otro modo la obligatoriedad de la enclisis pronominal en tales casos.

En el español normativo, el imperativo de primera persona del plural suprime la $s$ del morfema mos cuando le sigue el pronombre personal nos: / acostemos + nos / [ acostémonos ]. Tradicionalmente se ha querido explicar este fenómeno en términos fonéticos, argumentándose, por lo general, que se trata de la disimilación de eses (Cuervo, 1964: 490; Boyd-Bowman, 1960: 166; Kany, 1951: 175). Esta explicación parece inaceptable por varias razones. En primer lugar, no hay disimilación en casos como / acostemos + los / [ acostémoslos ]. En segundo lugar, si atribuimos la supresión de $s$ a la disimilación, nos veremos obligados a buscarle otra explicación a la elisión de $d$ en / sentad + os / [ sentáos ] a pesar de que en ambos casos pareciera tratarse de un mismo fenómeno.

La regla que suprime la consonante final en estos casos no es una regla fonológica, sino una regla morfológica y se aplica precisamente cuando el imperativo va seguido de un pronombre reflexivo. Para el español normativo peninsular podríamos formalizar la regla más o menos así:

(3) $\mathrm{C} \rightarrow \phi /\left[\mathrm{X}[+\right.$ imperativo $] \mathrm{Y}\left[\begin{array}{c}+ \text { plural } \\ -3 \text { forma }\end{array}\right]\left[\begin{array}{l}+ \text { pro } \\ + \text { ref }\end{array}\right]$

El rasgo [ -3 forma ] es necesario para evitar que la regla se aplique, por ejemplo, a / vayan + se / *[ váyase ]. En América, puesto que no se emplean las formas correspondientes a la persona vosotros, el rasgo [ -3 forma ] se cambiaría por [ +1 forma $]$. En ambos casos la función del rasgo es la misma.

En la gramática del español costarricense, la regla (3) parece haber sufrido una modificación, puesto que aquí no sólo se dice vámonos, levantémonos, etc., sino también digámoles, matémolo, etc. Es decir, aquí se suprime la consonante final del imperativo incluso cuando el pronombre que sigue no es reflexivo. Se ha incrementado el número de casos en que puede aparecer el alomorfo mo, por lo que parece tratarse de un ejemplo claro de extensión o polarización analógica. Si reformulamos la regla (3) para que se ajuste a la realidad lingüística de Costa Rica, quedará así:

(4) $\mathrm{C} \rightarrow \phi /\left[\mathrm{X}[+\right.$ imperativo $] \mathrm{Y}\left[\begin{array}{l}\overline{+ \text { plural }} \\ +1 \text { forma }\end{array}\right][+$ pro $]$

Vemos que, efectivamente, esta extensión analógica se refleja en la simplicación del índice estructural de la regla, puesto que hemos tenido que omitir el rasgo [ + ref ].

En vista de lo anterior, no deja de ser interesante otro cambio que se ha establecido ya en algunos sectores de la población de Costa Rica (y de otros muchos lugares, desde luego): córran$\mathrm{se}>$ córransen $>$ córrasen, diganme $>$ diganmen $>$ digamen, etc. Es decir, ahora también se está suprimiendo la consonante final de los imperativos [ + 3 forma ]. Algunos dialectólogos creen que en estos casos la $n$ final no se elide, sino que se metatetiza. Este punto de vista me parece, sin em- 
bargo, insostenible. En primer lugar, formas intermedias como córransen indican que no se trata de metatetizar la $n$ original, sino de añadir otra. En segundo lugar, las formas pronominales men, len, sen aparecen en contextos en los que de ningún modo cabría atribuirlas a la metátesis de la n: tendrán que irsen.

Aunque este cambio todavia no ha afectado todas las formas posibles (por ejemplo, que yo sepa en Costa Rica aún no se dice mátelon como en algunas regiones) los dialectos en los cuales se va consolidando parecen apuntar hacia una nueva versión de la regla (4).$$
\mathrm{C} \rightarrow \phi /[\mathrm{X}[+ \text { imperativo }] \mathrm{Y}[\overline{+ \text { plural }}][+ \text { pro }]
$$

Nuevamente se ve cómo la extensión de la regla se reflejaría en la simplificación del índice estructural. Parece estar en vías de perderse el rasgo
[ +1 forma]. Este análisis del problema es, desde luego, bastante tentativo. Pero tiene la ventaja, nada despreciable, de que da cuenta de la supresión de la consonante final de formas como matémolo y córrasen por medio de una sola regla. Para que fuese preferible la postulación de dos reglas diferentes, tendría que haber razones muy convincentes de que se trata de dos fenómenos distintos.

En algunos dialectos (por ejemplo, en ciertas modalidades mexicanas) se ha eligido el camino de la nivelación. En estas variantes la $s$ final de formas como vamos, matemos, etc., no se elide ni siquiera cuando sigue un clítico reflexivo: vámosnos, matémosmos, etc. Puesto que ya no hay alternancia alguna (entre los alomorfos - mos y $-m o$ ) se dice que la nivelación ha sido absoluta.

El cambio estructural no sólo se ha simplificado, sino que se ha perdido, y por tanto regla también.

\section{NOTAS}

1 Actualmente hay una tendencia hacia la separación del componente morfofonológico en dos componentes diferentes, lo cual no tiene, me parece, implicaciones importantes para este artículo.

2 Esta formalización es probablemente tosca pero puede servir como una primera aproximación. Por ejem- plo aunque en algunas regiones se dice mátelon, sáquelan, etc., me parece sumamente improbable que se llegue a decir mátenon por mátennos. Si por ejemplo, los imperativos [ +3 forma] llegaran a suprimir la consonante final delante de todos los clíticos menos el que corresponde a la primera persona del plural, esto tendría que explicitarse en la regla.

\section{Bibliografía}

BOY-BOWMAN, Peter (1960). El habla de guanajuato México: Imprenta Universitaria.

CUEKVO, Rufino José (1964). Notas a la gramática de la lengua castellana de Andrés Bello. Buenos Aires: Editorial Sopena Argentina, S.A.

HOENIGSWALD, Henry (1963/1966). "Are there Universals of Linguistic Change?" en Uni- versals of Languaje (compilador: Joseph Greenberg). Cambridge: The M.I.T. Press.

KANY, Charles (1945/1951). American Spanish Syntax. Chicago: University of Chicago Press.

KIPARSKY, Paul (1968). "Linguistic Universals and Linguistic Change" en Universals in Linguistic Theory /Compiladores: Emmon Bach y Robert T. Harms). Nueva York: Holt, Rinehart and Winston. 


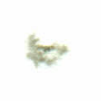

